\title{
IMPACT OF CHRONIC KIDNEY DISEASE ON OUTCOME OF PERCUTANEOUS CORONARY INTERVENTION IN ACUTE CORONARY SYNDROME
}

\author{
Hanan Radwan, ${ }^{I}$ Abdelhakem Selem, ${ }^{\text {Ii }}$ Yaser Ammarii And Naser Mahdi. ${ }^{\text {Iv }}$ \\ I-Lecturer of Cardiology, Faculty of Medicine, Zagazig University, Egypt. And Consultant of Cardiology, King Faisal \\ Specialist Hospital, KSA. \\ II-Assistant Professor of Cardiology, Faculty of Medicine, Zagazig University, Egypt. And Consultant of Cardiology, \\ Ibn Sina National Medical College Hospital, KSA. \\ III-Assistant Professor of Internal Medicine, Medical Research Institute, Alexandria University, Egypt. And Consultant \\ of nephrology, New Jeddah Clinic Hospital, KSA. \\ IV-Consultant of Cardiology, Interventional Cardiology Department, King Faisal Specialist Hospital, KSA.
}

\begin{abstract}
Objectives: We aim to study the impact of chronic kidney disease (CKD) on short and long term outcome of percutaneous coronary intervention (PCI) in patients with acute coronary syndrome (ACS).

BACKGROUND: Renal impairment (RI), even if mild, is a known independent predictor of cardiovascular events, but the effects of various grades of RI on PCI outcomes is not well established.

Methods: 427 patients with ACS treated within 24 hours with PCI were subjected to ECG, echocardiography, repeated measurements of cardiac troponin I (cTNI), cardiac isoenzyme of creatine kinase (CK-MB), serum creatinine and estimation of creatinine clearance $(\mathrm{eCrCl})$ by Cockcroft, Gault equation which classified patients into 4 groups as per $\mathrm{K} / \mathrm{DOQI}$ guidelines. Major adverse cardiovascular events (MACE) comprising death, new myocardial infarction (MI) and target vessel revascularization (TVR) were recorded during early (30 days) and late (up to 5 years) follow up period.

RESULTS: Advanced RI was associated with a significant increase in number of affected vessels, percentage of $\mathrm{C}$ type lesions and a significant decrease in culprit artery TIMI flow. $\mathrm{eCrCl}$ correlated positively with $\mathrm{EF}$ and negatively with number of vessels affected and number of late MACE. Patients with advanced RI had an increased risk of TVR in first month after PCI and an increased risk of death, MI and total MACE on long-term follow up.

Conclusion: CKD is associated with an increased need for TVR during the first 30 days, and increased death, MI and total MACE during later follow up in PCI treated ACS patients.

Key Words: Acute myocardial infarction, Acute coronary syndrome, Coronary angioplasty, Contrast agents, Chronic kidney disease.

\section{INTRODUCTION}

$\mathrm{t}$ is well known that impairment of renal function (RF) is an independent risk factor for cardiovascular disease (CVD) events, even if it is mild.(1-4) Among ACS patients, CKD is an independent predictor of MACE, and is associated with less frequent use of proven medical therapies.(5-7) Despite the increased incidence of bleeding and stent thrombosis in PCI treated CKD patients presenting with $\mathrm{ACS},(8,9)$ subjects with more advanced grades of renal dysfunction were generally excluded from clinical trials evaluating new CVD therapeutics. $(10,11)$ The impact of CKD on mortality and morbidity in ACS patients is apparently underappreciated(12) and the optimal therapeutic modality in CKD patients presenting with ACS has not been well defined.(13) Therefore, we studied the impact of various grades of CKD, including end stage renal disease (ESRD), on the short and long term outcome of PCI done for a cohort of ACS patients.

\section{MATERIALS AND METHODS}

This study was conducted in 3 referral centers in Jeddah, KSA: King Faisal Specialist Hospital, Ibn Sina National Medical College Hospital and New Jeddah Clinic Hospital. It enrolled 427 consecutive patients presenting with ACS [214

patients with unstable angina (UA), 144 patients with non ST segment elevation myocardial infarction (NSTEMI) and 69 patients with St segment myocardial infarction (STEMI)] treated with PCI within 24 hours over the period from January 2008 to June 2013. An informed written consent was obtained from each patient according to the declaration of Helsinki and the ethical committee of all included hospitals. All patients were subjected to full history taking, clinical examination, ECG and echocardiograph to assess left ventricular ejection fraction (LVEF). Diabetes mellitus, hypertension, dyslipidemia, stable angina, unstable angina, acute coronary syndrome and heart failure were defined according to the American Heart Association definitions for measuring the clinical management and outcomes of patients with ACS.(14) Laboratory studies included CBC, blood urea, serum creatinine, electrolytes, fasting blood sugar and lipid profile. Serum cTNI and CKMB were recorded on admission and every 8 hours during hospital admission time. The maximum values for cTNI and CK-MB were used for comparisons. The $\mathrm{eCrCl}$, based on serum creatinine value on admission, was calculated using the Cockcroft and Gault equation.(15) It classified patients into 4 groups as per Kidney Disease Outcomes Quality
\end{abstract}


Initiative (K/DOQI) guidelines on classification of CKD:(16)

- Group 1 (Normal RF): eCrCl $>90 \mathrm{ml} / \mathrm{min}$.

- Group 2 (Mild RI): eCrCl 60 - 89 ml/min (equivalent to $\mathrm{K} / \mathrm{DOQI} \mathrm{CKD}$ stage 2).

- Group 3 (Moderate RI): eCrCl 30 - $59 \mathrm{ml} / \mathrm{min}$ (equivalent to K/DOQI CKD stage 3 ).

Group 4 (Severe RI): eCrCl $<30 \mathrm{ml} / \mathrm{min}$, or treatment with regular haemodialysis (equivalent to K/DOQI CKD stages 4 and 5).

The protocol of patient diagnosis and management was based on the 2007 Focused Update of the ACC/AHA/SCAI 2005 Guideline Update for PCI,(17) and the following updates. $(18,19)$ Angiographic analysis for assessment of severity of coronary stenosis was done by two observers using visual assessment. The number of diseased coronary arteries was defined by the number of major coronary arteries with luminal diameter stenosis $>70 \%$ (or $\geq 50 \%$ stenosis in the left main coronary artery). The culprit artery was defined as the vessel responsible for the ACS. The culprit artery TIMI flow grading system was used for semi quantitative evaluation of myocardial perfusion before and after PCI.(20) One to three intra-coronary stents were inserted in most patients; they were mostly of the drug eluting types. The morphologic types of the coronary stenotic lesions were recorded according to the modified ACC/AHA classification of the primary target stenosis.(21) PCI procedural success was defined as successful stent placement at the desired position with $<30 \%$ residual stenosis.(22) Prevention of contrast- induced nephropathy (CIN) was implemented according to the European Society of Urogenital Radiology guidelines,(23) including assessment of baseline $\mathrm{RF}$, adequate hydration, use of the minimal possible dose of low osmolar agents and occasionally the administration of $\mathrm{N}$ acetylcysteine. Patients with baseline serum creatinine $>1.8 \mathrm{mg} / \mathrm{dl}$ and not on dialysis were given the dye in one third dilution and put on IV isotonic saline at a rate of $1 \mathrm{ml} / \mathrm{Kg} /$ hour for 6 hours before and 6 hours after angiography. $(24,25)$ Daily monitoring of urea, creatinine and fluid chart was employed for 5 days post- contrast injection and dialysis was instituted if indicated. CIN was defined as an impairment in $\mathrm{RF}$ (an increase in serum creatinine $>0.5 \mathrm{mg} / \mathrm{dl}$ or $>25 \%$ compared to baseline) occurring within 3 days following intravascular administration of contrast media, in absence of alternative etiology.(26) MACE were defined as the combined end point of death (of cardiac or procedure related origin), newly developed myocardial infarction, and clinically driven target vessel revascularization (TVR).(27) TVR was defined as repeat PCI or CABG (coronary artery bypass graft) performed for revascularization of a stenosis in the vessel originally treated. Restenosis was defined as a luminal diameter stenosis $>50 \%$ at follow up. MACE were subdivided into early MACE (recorded during the initial hospital stay and within one month from PCI) and late MACE (during the follow up which was done monthly and included repeat clinical and laboratory evaluation, medication review and, if indicated, TVR). The follow up period ranged from 39 to 70 months with an average of 49 months. Patients who presented with cardiogenic shock, acute renal failure, those in whom PCI was unsuccessful and those lost from follow up in less than 3 years were excluded from the study.

Statistical analysis was performed with SPSS 13.0 statistical package. Continuous data were expressed as mean \pm standard deviation (SD) and categorical data as percentages. Associations between degree of renal impairment and other variables were assessed using ANOVA for continuous variables and $\mathrm{Chi}$ square test for categorical variables. Correlation coefficient (r) was used to study the relation between 2 continuous variables. The composite end- point (MACE) was determined using the Kaplan-Meyer survival method then time-to-event analysis was conducted using the log-rank test. A $\mathrm{P}$ value < 0.05 was considered statistically significant. A P value $<0.01$ was considered as highly significant.

\section{RESULTS}

The baseline characteristics of the 4 study groups (Table I) showed no statistically significant difference regarding sex, prevalence of diabetes mellitus, hypertension and dyslipidemia and mean duration of follow up. The patients with moderate and severe RI had statistically significant older age and lower body mass index. The use of antiplatelets of the GPIIa/IIIb inhibitor group was significantly less in patients with more advanced degrees of RI.

There was no statistically significant difference between the 4 study groups regarding the maximum levels of CK-MB and cTNI before and after PCI (Table II-A). The mean values of the maximal levels of both cardiac markers declined after PCI in all study groups (Table II-B), but the decline was statistically significant only for CKMB in patients with normal RF.

The mean number of vessels affected was statistically higher in patients with more advanced RI (Table III). So, instances of triple vessel disease showed a significant clustering in patients 
with severe RI (Table IV). The grade of culprit artery TIMI flow following PCI and the percent dilatation of the culprit lesion (Table III) were both significantly lower in patients with higher grades of RI. No statistically significant difference in the number of stents inserted in the different groups was observed. Over the whole study cohort, we found a statistically significant positive correlation between $\mathrm{eCrCl}$ and both $\mathrm{EF}$ and percent dilataion of the most stenotic lesion (Table V). There was a statistically significant negative correlation between $\mathrm{eCrCl}$ and both the number of vessels affected and the number of late MACE.

Table (VI) and figure (1) reveal that type A lesions (most mild) were relatively more frequently encountered in patients with normal RF ormild RI compared with patients with moderate and severe RI. The reverse was true for type C lesions (most severe) which were relatively more frequently seen in patients with more advanced CKD.

The need for TVR was significantly more frequent in patients with severe RI compared to other groups (Table VII-A). Death, MI and total early MACE were not significantly different among the study groups. A highly significant increase in frequencies of late (up to 5 years) death, MI and total late MACE in patients with severe RI was observed compared to other groups (Table VII-B). Log rank test applied for comparison of Kaplan Meyer curves of MACE free survival in different study groups (Figure 2) showed a statistically significant steady decline in survival with each drop in the RF sector. $(\mathrm{X} 2=15.84, \mathrm{P}=0.001)$

Table (I): Baseline Characteristics, Use of GPIIb/IIIa inhibitors and Follow Up Duration of the Study Patients

\begin{tabular}{|c|c|c|c|c|c|c|}
\hline & $\begin{array}{l}\text { Normal } \\
\text { Renal } \\
\text { Function } \\
(\mathrm{n}=165)\end{array}$ & $\begin{array}{c}\text { Mild } \\
\text { Renal } \\
\text { Impairment } \\
(\mathrm{n}=109)\end{array}$ & $\begin{array}{l}\text { Moderate } \\
\text { Renal } \\
\text { Impairment } \\
(\mathrm{n}=115)\end{array}$ & $\begin{array}{c}\text { Severe } \\
\text { Renal } \\
\text { Impairment } \\
(\mathrm{n}=38)\end{array}$ & $\begin{array}{c}\text { Test } \\
\text { Statistic }\end{array}$ & $\mathbf{P}$ \\
\hline $\begin{array}{c}\text { Sex } \\
(\text { Male No, \%) }\end{array}$ & $\begin{array}{c}133 \\
(80.6 \%) \\
\end{array}$ & $\begin{array}{c}86 \\
(78.9 \%) \\
\end{array}$ & $\begin{array}{c}84 \\
(73 \%) \\
\end{array}$ & $\begin{array}{c}25 \\
(65.8 \%) \\
\end{array}$ & $\chi^{2}=5.11$ & $\begin{array}{l}0.164 \\
\text { (NS) }\end{array}$ \\
\hline $\begin{array}{c}\text { Age } \\
\text { (Years) }\end{array}$ & $\begin{array}{r}56.5 \\
\pm 9.4 \\
\end{array}$ & $\begin{array}{r}58.4 \\
\pm 7.8 \\
\end{array}$ & $\begin{array}{c}60.3 \\
\pm 11.4 \\
\end{array}$ & $\begin{array}{l}58.3 \\
\pm 10 \\
\end{array}$ & $F=3.509$ & $\begin{array}{l}0.015 \\
(\mathrm{~S})\end{array}$ \\
\hline $\begin{array}{c}\text { Body Mass } \\
\text { Index }\left(\mathrm{Kg} / \mathrm{m}^{2}\right)\end{array}$ & $\begin{array}{l}28.4 \\
\pm 5\end{array}$ & $\begin{array}{r}24.9 \\
+3.6 \\
\end{array}$ & $\begin{array}{r}25.1 \\
\pm 4.5 \\
\end{array}$ & $\begin{array}{l}24.5 \\
\pm 3 \\
\end{array}$ & $F=21$ & $\begin{array}{c}<0.001 \\
(\mathrm{HS})\end{array}$ \\
\hline $\begin{array}{l}\text { Diabetes } \\
\text { Mellitus } \\
\end{array}$ & $\begin{array}{c}80 \\
(48.5 \%) \\
\end{array}$ & $\begin{array}{c}55 \\
(50.5 \%) \\
\end{array}$ & $\begin{array}{c}65 \\
(56.5 \%) \\
\end{array}$ & $\begin{array}{c}18 \\
(47.4 \%) \\
\end{array}$ & $\chi^{2}=2.034$ & $\begin{array}{l}0.565 \\
\text { (NS) }\end{array}$ \\
\hline Hypertension & $\begin{array}{c}98 \\
(59.4 \%)\end{array}$ & $\begin{array}{c}74 \\
(67.9 \%)\end{array}$ & $\begin{array}{c}79 \\
(68.7 \%)\end{array}$ & $\begin{array}{c}24 \\
(63.2 \%)\end{array}$ & $\chi^{2}=3.334$ & $\begin{array}{l}0.343 \\
\text { (NS) }\end{array}$ \\
\hline Dyslipidemia & $\begin{array}{c}118 \\
(71.5 \%)\end{array}$ & $\begin{array}{c}79 \\
(72.5 \%)\end{array}$ & $\begin{array}{c}87 \\
(75.7 \%)\end{array}$ & $\begin{array}{c}21 \\
(55.3 \%)\end{array}$ & $\chi^{2}=5.93$ & $\begin{array}{l}0.115 \\
\text { (NS) }\end{array}$ \\
\hline $\begin{array}{c}\text { Use of } \\
\text { GPIIb/IIIa } \\
\text { inhibitors }\end{array}$ & $\begin{array}{c}101 \\
(61.2 \%)\end{array}$ & $\begin{array}{c}76 \\
(69.7 \%)\end{array}$ & $\begin{array}{c}54 \\
(47 \%)\end{array}$ & $\begin{array}{c}17 \\
(44.7 \%)\end{array}$ & $\chi^{2}=15.359$ & $\begin{array}{c}0.002 \\
\text { (HS) }\end{array}$ \\
\hline $\begin{array}{l}\text { Follow up } \\
\text { Duration } \\
\text { (Months) }\end{array}$ & $\begin{array}{r}49.2 \\
\pm 6.7\end{array}$ & $\begin{array}{r}50.2 \\
\pm 7.2\end{array}$ & $\begin{array}{r}47.7 \\
\pm 6.6\end{array}$ & $\begin{array}{r}48.7 \\
\pm 8.7\end{array}$ & $\mathrm{~F}=2.49$ & $\begin{array}{l}0.06 \\
\text { (NS) }\end{array}$ \\
\hline
\end{tabular}


Table (II): Maximum CK-MB and Troponin before and after PCI

(A) Comparison of the values among different renal function groups

\begin{tabular}{|c|c|c|c|c|c|c|}
\hline & $\begin{array}{c}\text { Normal } \\
\text { Renal } \\
\text { Function } \\
(\mathrm{n}=165)\end{array}$ & $\begin{array}{c}\text { Mild } \\
\text { Renal } \\
\text { Impairment } \\
(\mathrm{n}=109)\end{array}$ & $\begin{array}{c}\text { Moderate } \\
\text { Renal } \\
\text { Impairment } \\
(\mathrm{n}=115)\end{array}$ & $\begin{array}{c}\text { Severe } \\
\text { Renal } \\
\text { Impairment } \\
(\mathrm{n}=38)\end{array}$ & $\mathbf{F}$ & $\mathbf{P}$ \\
\hline $\begin{array}{l}\text { Maximum CK- } \\
\text { MB before PCI }\end{array}$ & $\begin{array}{c}466.7 \\
+962.4 \\
\end{array}$ & $\begin{array}{c}525.7 \\
+992.4 \\
\end{array}$ & $\begin{array}{c}501.9 \\
+1114.9 \\
\end{array}$ & $\begin{array}{r}457 \\
+373.5 \\
\end{array}$ & 0.1 & $\begin{array}{l}0.96 \\
\text { (NS) }\end{array}$ \\
\hline $\begin{array}{c}\text { Maximum CK- } \\
\text { MB } \\
\text { after PCI }\end{array}$ & $\begin{array}{c}219.4 \\
\pm 286.4\end{array}$ & $\begin{array}{c}363.1 \\
\pm 714.4 \\
\end{array}$ & $\begin{array}{c}347.6 \\
\pm 786.7 \\
\end{array}$ & $\begin{array}{c}319.4 \\
\pm 276.3 \\
\end{array}$ & 1.769 & $\begin{array}{c}0.152 \\
\text { (NS) }\end{array}$ \\
\hline $\begin{array}{c}\text { Maximum cTNI } \\
\text { before PCI }\end{array}$ & $\begin{array}{c}2.92 \\
+23.8 \\
\end{array}$ & $\begin{array}{r}1.65 \\
+3.1 \\
\end{array}$ & $\begin{array}{r}4.5 \\
+22.7 \\
\end{array}$ & $\begin{array}{r}2.44 \\
+3.6 \\
\end{array}$ & 0.433 & $\begin{array}{l}0.73 \\
(\mathrm{NS})\end{array}$ \\
\hline $\begin{array}{c}\text { Maximum cTNI } \\
\text { after PCI }\end{array}$ & $\begin{array}{c}0.8 \\
+1.3 \\
\end{array}$ & $\begin{array}{r}1.25 \\
+2.1 \\
\end{array}$ & $\begin{array}{c}2.52 \\
+16.3\end{array}$ & $\begin{array}{l}1.81 \\
\pm 3\end{array}$ & 0.943 & $\begin{array}{l}0.42 \\
(\mathrm{NS})\end{array}$ \\
\hline
\end{tabular}

CK-MB: cardiac isoenzymes of creatine kinasecTNI: cardiac troponin I

(B) Comparison of the values before and after PCI within each renal function group

\begin{tabular}{|c|c|c|c|c|c|}
\hline & & Before PCI & After PCI & $\mathrm{T}$ & $\mathrm{P}$ \\
\hline \multirow{2}{*}{$\begin{array}{l}\text { Normal Renal } \\
\text { Function } \\
(\mathrm{n}=165)\end{array}$} & CK-MB & $\begin{array}{c}466.7 \\
+962.4 \\
\end{array}$ & $\begin{array}{c}219.4 \\
\pm 286.4\end{array}$ & 3.163 & $\begin{array}{c}0.002 \\
(\mathrm{HS})\end{array}$ \\
\hline & cTNI & $\begin{array}{c}2.92 \\
\pm 23.8\end{array}$ & $\begin{array}{c}0.8 \\
\pm 1.3\end{array}$ & 1.141 & $\begin{array}{l}0.255 \\
\text { (NS) }\end{array}$ \\
\hline \multirow{2}{*}{$\begin{array}{c}\text { Mild } \\
\text { Renal } \\
\text { Impairment } \\
(\mathrm{n}=109)\end{array}$} & CK-MB & $\begin{array}{c}525.7 \\
\pm 992.4\end{array}$ & $\begin{array}{c}363.1 \\
\pm 714.4\end{array}$ & 1.388 & $\begin{array}{c}0.167 \\
\text { (NS) }\end{array}$ \\
\hline & cTNI & $\begin{array}{r}1.65 \\
\pm 3.1 \\
\end{array}$ & $\begin{array}{r}1.25 \\
\pm 2.1\end{array}$ & 1.106 & $\begin{array}{l}0.27 \\
(\mathrm{NS})\end{array}$ \\
\hline \multirow{2}{*}{$\begin{array}{c}\text { Moderate } \\
\text { Renal } \\
\text { Impairment } \\
(\mathrm{n}=115)\end{array}$} & CK-MB & $\begin{array}{c}501.9 \\
+1114.9 \\
\end{array}$ & $\begin{array}{r}347.6 \\
\pm 786.7 \\
\end{array}$ & 1.213 & $\begin{array}{l}0.227 \\
\text { (NS) }\end{array}$ \\
\hline & cTNI & $\begin{array}{r}4.5 \\
+22.7\end{array}$ & $\begin{array}{c}2.52 \\
\pm 16.3\end{array}$ & 0.762 & $\begin{array}{c}0.447 \\
(\mathrm{NS})\end{array}$ \\
\hline \multirow{2}{*}{$\begin{array}{c}\text { Severe } \\
\text { Renal } \\
\text { Impairment } \\
(\mathrm{n}=38)\end{array}$} & CK-MB & $\begin{array}{c}457 \\
+373.5 \\
\end{array}$ & $\begin{array}{c}319.4 \\
\pm 276.3\end{array}$ & 1.825 & $\begin{array}{l}0.072 \\
(\mathrm{NS})\end{array}$ \\
\hline & cTNI & $\begin{array}{r}2.44 \\
+3.6\end{array}$ & $\begin{array}{l}1.81 \\
\pm 3\end{array}$ & 0.828 & $\begin{array}{c}0.411 \\
\text { (NS) }\end{array}$ \\
\hline
\end{tabular}

CK-MB: cardiac isoenzymes of creatine kinase cTNI: cardiac troponin I 
Table (III): Angiographic and PCI Data

\begin{tabular}{|c|c|c|c|c|c|c|}
\hline & $\begin{array}{c}\text { Normal } \\
\text { Renal } \\
\text { Function } \\
(\mathrm{n}=165)\end{array}$ & $\begin{array}{c}\text { Mild } \\
\text { Renal } \\
\text { Impairment } \\
(\mathrm{n}=109)\end{array}$ & $\begin{array}{l}\text { Moderate } \\
\text { Renal } \\
\text { Impairment } \\
(\mathrm{n}=115)\end{array}$ & $\begin{array}{c}\text { Severe } \\
\text { Renal } \\
\text { Impairment } \\
(\mathrm{n}=38)\end{array}$ & $\mathbf{F}$ & $\mathbf{P}$ \\
\hline $\begin{array}{c}\text { Number of } \\
\text { Vessels Affected }\end{array}$ & $\begin{array}{r}1.255 \\
\pm 0.56 \\
\end{array}$ & $\begin{array}{r}1.34 \\
\pm 0.6 \\
\end{array}$ & $\begin{array}{r}1.313 \\
\pm 0.54 \\
\end{array}$ & $\begin{array}{r}1.579 \\
\pm 0.79 \\
\end{array}$ & 3.194 & $\begin{array}{l}0.023 \\
(\mathrm{~S})\end{array}$ \\
\hline $\begin{array}{c}\text { Number of } \\
\text { Stents Inserted }\end{array}$ & $\begin{array}{r}1.582 \\
\pm 0.83 \\
\end{array}$ & $\begin{array}{r}1.844 \\
\pm 0.92 \\
\end{array}$ & $\begin{array}{r}1.722 \\
\pm 0.95 \\
\end{array}$ & $\begin{array}{r}1.684 \\
\pm 0.81 \\
\end{array}$ & 1.964 & $\begin{array}{l}0.119 \\
(\mathrm{NS})\end{array}$ \\
\hline $\begin{array}{c}\text { Culprit Artery } \\
\text { TIMI Flow }\end{array}$ & $\begin{array}{r}1.533 \\
\pm 0.69 \\
\end{array}$ & $\begin{array}{l}1.752 \\
\pm 0.76 \\
\end{array}$ & $\begin{array}{c}1.53 \\
\pm 0.74 \\
\end{array}$ & $\begin{array}{r}1.421 \\
\pm 076 \\
\end{array}$ & 3.027 & $\begin{array}{l}0.029 \\
(\mathrm{~S})\end{array}$ \\
\hline $\begin{array}{l}\text { Percent } \\
\text { dilatation of the } \\
\text { culprit lesion }\end{array}$ & $\begin{array}{c}91.33 \\
\pm 8.7\end{array}$ & $\begin{array}{l}92.84 \\
\pm 9.1\end{array}$ & $\begin{array}{l}88.35 \\
\pm 10.6\end{array}$ & $\begin{array}{l}85.53 \\
\pm 10.6\end{array}$ & 8.257 & $\begin{array}{l}<0.001 \\
\text { (HS) }\end{array}$ \\
\hline
\end{tabular}

Table (IV): Extent of Coronary Artery Involvement In Different Groups of Renal Function

\begin{tabular}{lcccc}
\hline Coronary & \multicolumn{4}{c}{ Renal function Group } \\
\hline Involvement & $\begin{array}{c}\text { Normal RF } \\
(\mathrm{n}=165)\end{array}$ & $\begin{array}{c}\text { Mild RI } \\
(\mathrm{n}=109)\end{array}$ & $\begin{array}{c}\text { Moderate RI } \\
(\mathrm{n}=115)\end{array}$ & $\begin{array}{c}\text { Severe RI } \\
(\mathrm{n}=38)\end{array}$ \\
\hline Single Vessel & 133 & 78 & 83 & 22 \\
& $(80.6 \%)$ & $(71.6 \%)$ & $(72.2 \%)$ & $(57.9 \%)$ \\
\hline Double Vessels & 22 & 26 & 28 & 11 \\
& $(13.3 \%)$ & $(23.9 \%)$ & $(24.3 \%)$ & $(28.9 \%)$ \\
\hline Triple Vessels & 10 & 5 & 4 & $5 \%$ \\
\hline * One patient has 4 vessel disease & $(6.1 \%)$ & $(4.6 \%)$ & $(3.5 \%)$ & $(13.2 \%)$ \\
$\chi^{2}=14.412$ & & & & \\
$\mathrm{P}=0.025$ (Significant) & & & &
\end{tabular}


Table (V): Some Statistical Correlations of Creatinine Clearance With Other Study Parameters

\begin{tabular}{lcc}
\hline Parameter Correlated with Creatinine Clearance & $\mathrm{P}$ & $<0.001$ \\
LVEF & 0.332 & $\mathrm{HS})$ \\
\hline Maximum CK-MB before PCI & 0.002 & $\begin{array}{c}0.97 \\
(\mathrm{NS})\end{array}$ \\
\hline Maximum CK-MB after PCI & -0.077 & $\begin{array}{c}0.113 \\
(\mathrm{NS})\end{array}$ \\
\hline Maximum cTNI before PCI & -0.01 & $\begin{array}{c}0.845 \\
(\mathrm{NS})\end{array}$ \\
\hline Maximum cTNI after PCI & -0.054 & $\begin{array}{c}0.265 \\
(\mathrm{NS})\end{array}$ \\
\hline Number of Vessels Affected & -0.099 & $\begin{array}{c}0.041 \\
(\mathrm{~S})\end{array}$ \\
\hline Number of Stents Inserted & -0.046 & $\begin{array}{c}0.279 \\
(\mathrm{NS})\end{array}$ \\
\hline Percent dilatation of the most stenotic lesion & 0.0034 & $\begin{array}{c}0.361 \\
(\mathrm{NS})\end{array}$ \\
\hline Number of Early MACE & 0.065 & $\begin{array}{c}0.179 \\
(\mathrm{NS})\end{array}$ \\
\hline Number of Late MACE & -0.232 & $<0.001$ \\
$(\mathrm{HS})$
\end{tabular}

LVEF: left ventricular ejection fraction.

PCI percutaneous intervention.

CK-MB: cardiac isoenzymes of creatine kinase.

cTNI: Cardiac troponin I.

MACE: Major Adverse cardiac events.

Table (VI): Frequency of Different Angiographic Types of Coronary Artery Lesions In Different Groups of Renal Function

\begin{tabular}{ccccc}
\hline \multirow{2}{*}{$\begin{array}{c}\text { Lesion } \\
\text { Type }\end{array}$} & $\begin{array}{c}\text { Normal RF } \\
(\mathrm{n}=165)\end{array}$ & $\begin{array}{c}\text { Mild RI } \\
(\mathrm{n}=109)\end{array}$ & $\begin{array}{c}\text { Moderate RI } \\
(\mathrm{n}=115)\end{array}$ & $\begin{array}{c}\text { Severe RI } \\
(\mathrm{n}=38)\end{array}$ \\
\cline { 2 - 5 } & 41 & 27 & 11 & 8 \\
$\mathrm{~A}$ & $(24.8 \%)$ & $(24.8 \%)$ & $(9.6 \%)$ & $(21.1 \%)$ \\
\hline B1 & 35 & 25 & 22 & 12 \\
& $(21.2 \%)$ & $(22.9 \%)$ & $(19.1 \%)$ & $(31.6 \%)$ \\
\hline B2 & 63 & 50 & 56 & 23 \\
& $(38.2 \%)$ & $(45.9 \%)$ & $(48 . \%)$ & $(60.5 \%)$ \\
\hline C & 65 & 44 & & 34 \\
& $(39.4 \%)$ & $(40.4 \%)$ & $(52.2 \%)$ & $(89.5 \%)$ \\
\hline
\end{tabular}

The frequencies are presented as number and (percentage).

Number: $\quad$ Number of patients who have at least one lesion of the specified type

Percentage: Percentage of the above number to the total number of patients within the specified renal function group

$\chi^{2}=17.918$

$\mathrm{P}=0.036$ (Significant) 
Table (VII): Major Adverse Cardiac Events (MACE)

At 30 Days and 5 Years After PCI

(A) Mean Number of Early MACE (Within 30 Days after PCI)

\begin{tabular}{|c|c|c|c|c|c|c|}
\hline Total 424 patients & $\begin{array}{c}\text { Normal } \\
\text { Renal } \\
\text { Function } \\
(\mathrm{n}=165)\end{array}$ & $\begin{array}{c}\text { Mild } \\
\text { Renal } \\
\text { Impairment } \\
(\mathrm{n}=109)\end{array}$ & $\begin{array}{c}\text { Moderate } \\
\text { Renal } \\
\text { Impairment } \\
(\mathrm{n}=115)\end{array}$ & $\begin{array}{c}\text { Severe } \\
\text { Renal } \\
\text { Impairment } \\
(\mathrm{n}=38)\end{array}$ & $\mathbf{F}$ & $\mathbf{P}$ \\
\hline Death & $\begin{array}{r}0.006 \\
\pm 0.08 \\
\end{array}$ & $\begin{array}{l}0.009 \\
\pm 0.1 \\
\end{array}$ & $\begin{array}{r}0.0 \\
\pm 0.0 \\
\end{array}$ & $\begin{array}{r}0.026 \\
\pm 0.16 \\
\end{array}$ & 0.976 & $\begin{array}{c}0.404 \\
(\mathrm{NS}) \\
\end{array}$ \\
\hline $\begin{array}{l}\text { Myocardial } \\
\text { Infarction }\end{array}$ & $\begin{array}{r}0.006 \\
\pm 1041 \\
\end{array}$ & $\begin{array}{r}0.018 \\
\pm 0.13 \\
\end{array}$ & $\begin{array}{r}0.17 \\
\pm 0.13 \\
\end{array}$ & $\begin{array}{r}0.053 \\
\pm 0.23 \\
\end{array}$ & 1.407 & $\begin{array}{l}0.24 \\
(\mathrm{NS}) \\
\end{array}$ \\
\hline $\begin{array}{c}\text { Target Vessel } \\
\text { Revascularization }\end{array}$ & $\begin{array}{r}0.012 \\
\pm 0.11 \\
\end{array}$ & $\begin{array}{l}0.009 \\
\pm 0.1 \\
\end{array}$ & $\begin{array}{r}0.017 \\
\pm 0.13 \\
\end{array}$ & $\begin{array}{r}0.079 \\
\pm 0.27 \\
\end{array}$ & 2.843 & $\begin{array}{c}0.037 \\
(\mathrm{~S})\end{array}$ \\
\hline $\begin{array}{l}\text { Total Early } \\
\text { MACE }\end{array}$ & $\begin{array}{r}0.024 \\
\pm 0.22 \\
\end{array}$ & $\begin{array}{r}0.037 \\
+0.27 \\
\end{array}$ & $\begin{array}{r}0.035 \\
+0.26 \\
\end{array}$ & $\begin{array}{r}0.158 \\
+0.59 \\
\end{array}$ & 2.197 & $\begin{array}{l}0.088 \\
(\mathrm{NS}) \\
\end{array}$ \\
\hline
\end{tabular}

(B) Mean Number of Late MACE (Up to 5 Years after PCI)

\begin{tabular}{|c|c|c|c|c|c|c|}
\hline Total 424 patients & $\begin{array}{l}\text { Normal } \\
\text { Renal } \\
\text { Function } \\
(\mathrm{n}=164)\end{array}$ & $\begin{array}{c}\text { Mild } \\
\text { Renal } \\
\text { Impairment } \\
(\mathrm{n}=108)\end{array}$ & $\begin{array}{l}\text { Moderate } \\
\text { Renal } \\
\text { Impairment } \\
(\mathrm{n}=115)\end{array}$ & $\begin{array}{c}\text { Severe } \\
\text { Renal } \\
\text { Impairment } \\
(\mathrm{n}=37)\end{array}$ & $\mathbf{F}$ & $\mathbf{P}$ \\
\hline Death & $\begin{array}{r}0.024 \\
\pm 0.15 \\
\end{array}$ & $\begin{array}{r}0.056 \\
\pm 0.23 \\
\end{array}$ & $\begin{array}{l}0.096 \\
\pm 0.3 \\
\end{array}$ & $\begin{array}{r}0.243 \\
\pm 0.43 \\
\end{array}$ & 8.24 & $\begin{array}{c}<0.001 \\
(\mathrm{HS}) \\
\end{array}$ \\
\hline $\begin{array}{l}\text { Myocardial } \\
\text { Infarction }\end{array}$ & $\begin{array}{c}0.03 \\
\pm 0.17 \\
\end{array}$ & $\begin{array}{r}0.056 \\
\pm 0.23 \\
\end{array}$ & $\begin{array}{r}0.148 \\
\pm 0.36 \\
\end{array}$ & $\begin{array}{r}0.108 \\
\pm 0.31 \\
\end{array}$ & 4.98 & $\begin{array}{l}0.002 \\
(\mathrm{HS}) \\
\end{array}$ \\
\hline $\begin{array}{c}\text { Target Vessel } \\
\text { Revascularization }\end{array}$ & $\begin{array}{r}0.049 \\
+0.22 \\
\end{array}$ & $\begin{array}{r}0.083 \\
+0.28 \\
\end{array}$ & $\begin{array}{c}0.07 \\
+0.26 \\
\end{array}$ & $\begin{array}{r}0.108 \\
+0.31 \\
\end{array}$ & 0.759 & $\begin{array}{l}0.218 \\
(\mathrm{NS}) \\
\end{array}$ \\
\hline Total Late MACE & $\begin{array}{r}0.104 \\
\pm 0.34 \\
\end{array}$ & $\begin{array}{r}0.194 \\
+0.44 \\
\end{array}$ & $\begin{array}{r}0.313 \\
+0.61 \\
\end{array}$ & $\begin{array}{r}0.459 \\
+0.69 \\
\end{array}$ & 7.548 & $\begin{array}{l}<0.001 \\
(\mathrm{HS})\end{array}$ \\
\hline
\end{tabular}

Figure 1. Frequency of different coronary lesions in each renal function group.

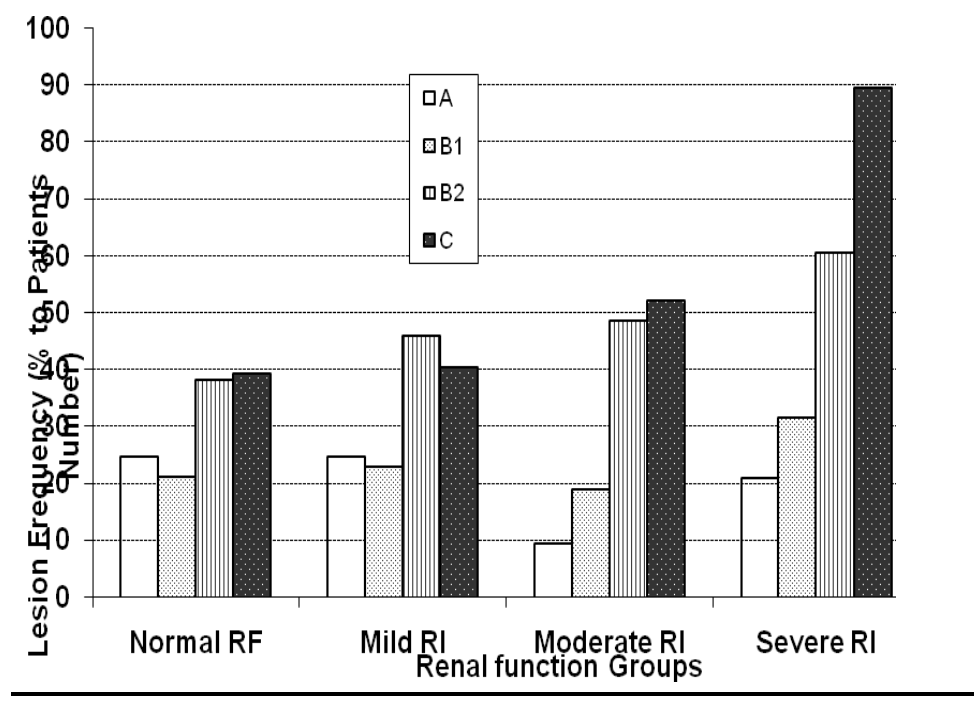




\section{Figure 2. Showing Caplan Mayer curve of late MACE after PCI.}

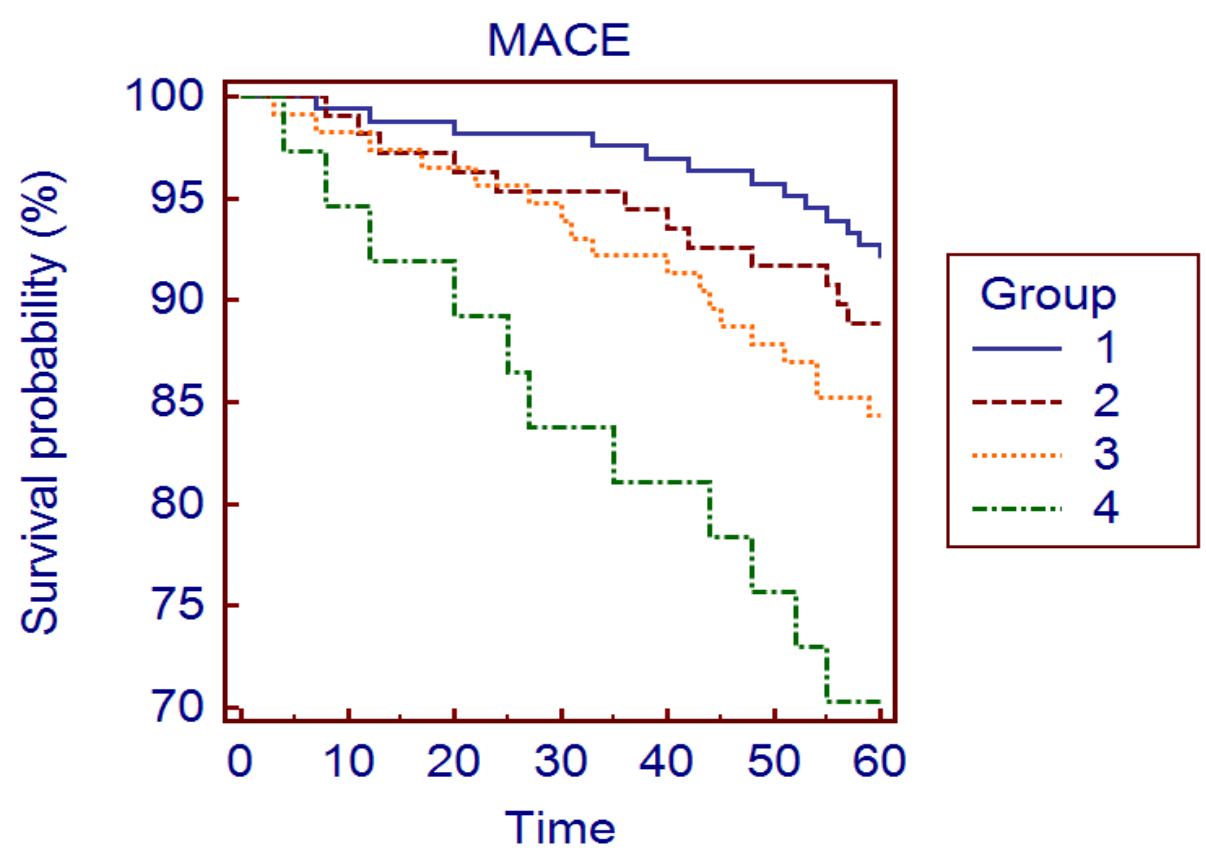

(Months)

MACE: Major Adverse Cardiac Events.

Group1: Normal renal function.

Group3: Moderate renal Impairment.

\section{DISCUSSION}

CKD is a worldwide public health problem with poor outcomes and high cost.(1) Individuals with CKD are more likely to die of CVD than to develop kidney failure.(28) RI has consistently been found to be an independent risk factor for CVD outcomes and all cause mortality in patients undergoing PCI; it appears that the increased risk is present even with mild reductions in kidney function.(1-4) ACS patients with RI have generally been systemically excluded from large cardiovascular trials.(29) It was perceived that PCI is inappropriately underutilized in ACS patients with concomitant RI.(30)

Therefore, with advances in PCI techniques and overall improvements in outcome, we decided to explore the impact of CKD on PCI outcome in a cohort of patients presenting with ACS. The categorization of the study patients was based on $\mathrm{eCrCl}$ at presentation as per K/DOQI guidelines.(16) This was similarly adopted in other studies addressing the effect of CKD on PCI outcome.(31,32) We included patients covering the whole spectrum of CKD, including ESRD patients. Patients with some degree of RI constituted 262/427 (61 \%) of the study population, which was very close to the ratio reported by Freeman et al (32) (62\%), but higher than the ratio reported by Inrig et al (33) $(40 \%)$ who excluded patients with severe $\mathrm{CKD}(\mathrm{eCrCl}<$ $30 \mathrm{ml} / \mathrm{min}$ ). Patients with baseline RI were significantly older (Table I), as reported in other studies. $(32,33)$

The significantly higher BMI in patients with baseline normal RF (Table I) may be explained by freedom from the wasting/cachexia syndrome in CKD patients which consists of anorexia, increased energy expenditure and decreased protein stores.(34) Many studies found diabetes mellitus and hypertension to be more prevalent in CKD patients compared to patients with normal RF. The association of CKD with dyslipidaemia was inconsistent. $(2,32,33,35)$ Patients with RI in the present study did not have more known baseline CVD risk factors (apart from RI itself). Even in studies revealing increased prevalence of DM, HTN and dyslipidaemia, the association of RI with increased MACE was independent of all other measured variables. $(2,32,33,35)$

The underutilization of cardiac medications and interventions in CKD patients presenting with ACS might be attributed to increased bleeding complications with the use of antiplatelets(32) and fear of CIN following coronary angiography(33). 
In the present study, the use of GPIIb/IIIa inhibitors was significantly less in patients with more advanced grades of RI. Winkelmayer et al(36) found that the use of aspirin is limited in elderly dialysis patients after AMI. The problem may be further compounded by blunted response to the antiplatelet agent within the uraemic milieu, as reported for clopidogrel(37). Shlipak et al(38) has found that RI is associated with lower use of PCI, antiplatelets and $\beta$-blockers both during hospitalization for AMI and after discharge.

The maximum values of CK-MB and cTNI before and after PCI encompassed a wide range with high standard deviations and so they showed no statistically significant differences among the 4 study groups (Table II-A). Also, we found no significant correlation between these biomarkers and $\mathrm{eCrCl}$ (Table $\mathrm{V}$ ). These results were concordant with those of $\mathrm{Li}$ et al(31) who found no significant relationship between status of RF and biomarkers of myocardium necrosis and presumed that acute STEMI caused such a severe damage to the myocardium that eliminated the effects of RF. Within each group, the values became lower after PCI, as expected (table II-B). The decline following PCI was statistically significant only for CK-MB in patients with normal RF. The absence of significant drop in these cardiac markers even after successful PCI may be explained by that RI per se increases serum CK-MB(39) and cardiac troponins(40). Also, comparing only the maximum levels of the biomarkers may be less sensitive for detection of the decline(40).

There was a significant increase in the number of vessels affected in patients with CKD compared to patients with normal RF, with the highest mean number of vessels affected observed in patients with severe RI (Table III). Consequently, patients with triple vessel affection tended to significantly cluster in the severe RI group (Table IV). All over the studied patients, the number of vessels affected showed a statistically significant negative correlation with baseline $\mathrm{eCrCl}$ (Table V). In accord with these results, Best et al(2) found triple vessel disease to be more frequent in patients with severe RI or patients on dialysis compared with patients with mild to moderate RI $(\mathrm{P}<0.001)$. Also, Gitt et al 2010(41) found that in patients with ACS, the prevalence of three vessels disease in patients with renal failure was $44.5 \%$ compared to $23.8 \%$ only in patients without.

The mean number of coronary stents inserted per case was not, however, higher in patients with severe RI compared with the other groups. Similarly, in the study of Best et al (2), the lowest
$\mathrm{CrCl}$ group had a greater number of diseased coronary vessels than the higher $\mathrm{CrCl}$ group, but had a similar number of vessels treated. These findings may reflect the difficulties in cannulation and stenting of the atherosclerotic/calcified coronary arteries in uraemic patients, who, even in the predialysis stage, are known to have increased arterial stiffness.(42) Coronary angiographic studies have revealed a high prevalence of calcified, tortuous and restenotic lesions in patients with RI (2).

Three other important indicators of the higher technical difficulty and lower procedural success of PCI performed in patients with more advanced grades of CKD in this study are: (1) the significantly lower post-dilatation culprit artery TIMI flow, (2) the significantly lower percent dilatation of the culprit lesion (Table III), and (3) the significant positive correlation between baseline $\mathrm{CrCl}$ and percent dilatation of the most stenotic lesion (Table V). These results were concordant with those of Appleby et al (43) who found that culprit artery TIMI flow was significantly lower in patients with $\mathrm{CrCl}<$ $60 \mathrm{ml} / \mathrm{min}$ compared to patients with $\mathrm{CrCl}>$ $60 \mathrm{ml} / \mathrm{min}$.

Freeman et al(32) found a significantly lower LVEF in patients with RI compared to patients without. In the present study, we found a statistically significant positive correlation between baseline $\mathrm{eCrCl}$ and LVEF (Table V). There is increasing evidence that uremic toxins adversely affect cardiac function (44). This may in turn adversely affect the outcome of PCI in uremic patients(45). Best et al (2) found a steady decline of LVEF across the 4 groups of RF that were classified according to baseline $\mathrm{CrCl}(>70$, $50-69,30-49,<30 \mathrm{ml} / \mathrm{min}$ ). Patients on dialysis were considered a separate group and they had a relatively increased EF (most probably reflecting a state of hyperdynamic circulation or hyperdynamic heart failure rather than improved myocardial function). However; in our study we excluded all patients on renal dialysis.

We applied the modified ACC/AHA classification of the primary target stenosis to characterize the morphologic types of the coronary lesions (21). This scheme recognizes type A lesions as the most amenable for successful dilatation $(>85 \%)$, with low risk of abrupt vessel closure. Type $\mathrm{C}$ lesions have the least possibility for successful dilatation $(<60 \%)$ and the highest risk of abrupt vessel closure. We found a statistically significant relative increase in type $\mathrm{C}$ lesions in patients with more advanced grades of RI (Table VI, Figure 1). Type A lesions, on the other hand, tended to 
cluster in patients with normal RF or mild RI. A similar finding was reported by Best et al (2). A similar finding was not detected in another study that excluded patients with serum creatinine $>1.8 \mathrm{mg} / \mathrm{dl}$ (46), denoting that this differential distribution of coronary artery lesions is probably present only with severe degrees of RI. Utilizing integrated backscatter intravascular ultrasound, Miyagi et al(47) found a decline in $\mathrm{eCrCl}$ to be significantly correlated with a decline in fibrous tissue content and an increase in fat content of the atheromatous plaques; changes which indicate plaque vulnerability and herald plaque rupture and AMI.

Although PCI is an effective therapy for ACS, it remains associated with high complications rate and poor long-term outcomes in patients with advanced CKD(48). The burden of post- PCI morbidity and mortality was expressed in this study as the MACE the composite end point of death, MI and clinically driven target vessel or target lesion revascularization (TVR or TLR). This is the most commonly used composite end point in cardiovascular research(49). Kahn JK et al (50) used the more inclusive term, TVR, which encompasses, in addition to TLR, interventions outside the target lesion performed to treat other atherosclerotic plaques as well as interventions done within the target lesion to treat neo-intimal hyperplasia resulting in in-stent restenosis. Although TLR may better reflect the effectiveness of the initial intervention, TVR better reflects the increased burden of coronary atherosclerosis in patients with various grades of RI compared to patients without.

PCI in patients with ESRD is associated with a 60-81\% incidence of restenosis(51). In the present study, patients with severe RI had higher incidence of clinically driven TVR within 30 days of PCI compared with other RF groups (Table VII-A). Difficulties in technique due to arterial wall stiffness (42) compounded with bleeding complications(41) afflicting the atheromatous wall might explain this.

During long-term follow up, the increased TVR instances in severe RI patients was not statistically significant compared to other RF groups, probably due to the relatively small percentage of these patients $(37 / 424=8.7 \%)$. Similar to the results of Gitt et $\mathrm{al}(41)$ and $\mathrm{Li}$ et al,(31) the risk of inhospital MI was not increased in patients with RI compared to patients without. However, these investigators found an increased risk of in-hospital mortality for patients with RI compared to patients without. Such an increased risk was not found in the present study.
We found a highly significant negative correlation between baseline $\mathrm{eCrCl}$ and number of late MACE (Table V). The particular events that occurred at a significantly higher rate in patients with more severe degrees of RI during long-term follow up were death and MI (Table VIIB), leading to a significantly higher incidence of total rate MACE and a significantly lower MACE free survival curve in patients with severe RI compared to the other groups (Figure 2). Na KY et al (52) reported survival curves in different CKD stages following PCI that were very close to our results. They found that the strongest predictors for mortality were CKD stage 5 and old age $(\mathrm{P}<0.001$ for both). Best et al (2) found a significantly increased risk of death and $\mathrm{MI}$ in patients with severe $\mathrm{RI}(\mathrm{eCrCl}<30 \mathrm{ml} / \mathrm{min}$ ) during one year interval following PCI. Similar to our results, the incidence of TVR was not increased in these patients. However, the actual incidence of restenosis in these patients is expected to be high(53) and so the significantly increased risk of death and MI during long term follow up found by Best et al and the present study may denote the presence of severe silent ischaemia.

\section{RESEARCH LIMITATIONS}

The effect of exposure to IV contrast used in PCI on RF was not specifically addressed in this study. However, occasional post-contrast injection rise in serum creatinine were only mild and recoverable with conservative measures like good hydration, pharmaco-vigilance for potentially nephrotoxic medications and occasional administration of acetylcysteine.(22)

Duration and etiology of CKD could not be precisely defined in some cases. Therefore, recent changes in RF at time of enrollment due to acute events could not be excluded and may make the baseline $\mathrm{eCrCl}$ not perfectly representing the basal level of CKD.

Creatinine based methods for estimation of glomerular filtration rate carry substantial degrees of inaccuracy(54). Finally, other outcome measures such as functional status and quality of life were not addressed.

\section{CONCLUSION}

Results of PCI in patients with severe RI remain suboptimal despite technological advances and increased use of coronary stents. These patients may be deprived the opportunity to receive GPIIb/IIIa inhibitors and other potentially beneficial medications. They have a significantly increased risk of TVR within one month of the index procedure and a significantly increased risk of MI, death and total MACE on the long term. Severe silent ischaemia may account for the 
increased cardiac mortality in ESRD patients even after successful PCI. This underscores the need for vigilant regular follow up of these patients for early recognition and management of coronary insufficiency.

Recognition of the excess risk burden of various grades of RI in ACS patients treated with PCI is essential for risk stratification and may assist in development of management strategies tailored to improve outcome. Further investigations and targeted randomized clinical trial are needed to define the best medical and/or interventional approach for management of ACS in patients with various stages of CKD.

\section{REFERENCES}

1) Sarnak MJ, Levey AS, Schoolwerth AC, Coresh J. Kidney disease as a risk factor for development of cardiovascular disease: a statement from the American Heart Association councils on kidney in cardiovascular disease, high blood pressure research, clinical cardiology, and epidemiology and prevention. Circulation 2003; 108: 2154- 69.

2) Best PJ, Lennon R, Ting HH. The impact of renal insufficiency on clinical outcomes in patients undergoing percutaneous coronary intervention. J Am Coll Cardiol 2002; 39: 1113- 119.

3) Goldsmith DJA, Covic A. Coronary artery disease in uremia: etiology, diagnosis, and therapy. Kidney Int 2001; 60: 2059- 78.

4) Parfrey PS, Foley RN. The clinical epidemiology of cardiac disease in chronic renal failure. J Am Soc Nephrol 1999; 10: 1606-15.

5) Reddan DN, Szczech L, Bhapkar MV, Moliterno DJ. Renal function, concomitant medication use and outcomes following acute coronary syndromes. Nephrol Dial transplant 2005; 20:2105- 12.

6) Shlipak MG, Heidenreich PA, Noguchi H. Association of renal insufficiency with treatment and outcomes after myocardial infarction in elderly patients. Ann Intern Med 2002; 137: 555- 62.

7) Widimsky P, Rychlik I. Renal disease and acute coronary syndrome. Heart 2010; 96: 86- 92.

8) Pitsavos C, Kourlaba G, Panagiotakos DB, Kogias Y, Mantas Y, Chrysohoou, C, Stefanadis C. Association of creatinine clearance and in-hospital mortality in patients with acute coronary syndromes: the greecs study. Circ J 2007; 71 (1): 914.

9) Zachee P, Vermylen J, Boogaerts MA. Hematologic aspects of endstage renal failure. Ann Hematol 1994; 69 (1): 33- 40.

10) Mann JF, Gerstein HC, Pogue J, Bosch J, Yusuf S. Renal insufficiency as a predictor of cardiovascular outcomes and impact of ramapril: the HOPE randomized trial. Ann Intern Med 2001; 134: 62936.

11) Pahor M, Shorr RI, Somes GW. Diuretic-based treatment and cardiovascular events in patients with mild renal dysfunction enrolled in the systolic hypertension in the elderly program. Arch Intern
Med 1998; 158: 1340- 5.

12) Reddan DN, Szczech L, Bhapkar MV, Moliterno DJ. Renal function, concomitant medication use and outcomes following acute coronary syndromes. Nephrol Dial Transplant 2005; 20:2105- 12.

13) Anavekar NS, Pfeffer MA. Cardiovascular risk in chronic kidney disease. Kidney Int 2004; (Suppl 92): S11- 5 .

14) Cannon CP, Brindis RG, Chaitman BR, Cohen DJ. 2013 ACCF/AHA Key Data Elements and Definitions for Measuring the Clinical Management and Outcomes of Patients with Acute Coronary Syndromes and Coronary Artery Disease: A Report of the American College of Cardiology Foundation/American HeartAssociation Task Force on Clinical Data Standards. Circulation 2013; 127 : 1052- 89.

15) Cockcroft DW, Gault MH. Prediction of creatinine clearance from serum creatinine. Nephron 1976; 16 : 31-41.

16) $\mathrm{K} / \mathrm{DOQI}$ clinical practice guidelines for chronic kidney disease: evaluation, classification, and stratification. Am J Kid Dis 2002: 39 (Suppl 1): S1266.

17) King SB, Smith SC, Hirshfeld JW, Jacobs AK. 2007 Focused Update of the ACC/AHA/SCAI 2005 Guideline Update for Percutaneous Coronary Intervention: a report of the American College of Cardiology/American Heart Association Task Force on Practice Guidelines. Circulation 2008; 117: 26195.

18) Kushner FG, Hand M, Smith SC, King SB. 2009 Focused Updates: ACC/AHA Guidelines for the Management of Patients With ST-Elevation Myocardial Infarction. Circulation 2009; 120:227306.

19) Levine GN, Bates ER, Blankenship JC. 2011 ACCF/AHA/SCAI Guideline for Percutaneous Coronary Intervention. J Am Coll Cardiol 2011; 58: e44- 122.

20) The TIMI Study Group. The Thrombolysis in Myocardial Infarction (TIMI) trial: phase I findings. N Engl J Med 1984; 33:523- 30.

21) Ellis SG, Vandormael MG, Cowley MJ, DiSciascio G. Coronary morphologic and clinical determinants of procedural outcome with angioplasty for multivessel coronary disease. Implications for patient selection. Circulation 1990; 82: 1193- 202.

22) Schuhlen. H, Kastrati A, Dirschinger J, Hausleiter J.Intracoronary stenting and risk for major adverse cardiac events during the first month. Circulation 1998; 98: 104- 11 .

23) Thomson HS, Morcos SK. Contrast media and the kidney:European Society of Urogenital Radiology (ESUR) guidelines. Br J Radiol 2003; 76: 513- 8.

24) Bruce RJ, Djamali A, Shinki K,et al. Background fluctuation of kidney function versus contrastinduced nephrotoxicity. AJR Am J Roentgenol 2009;192:711-718.

25) Pakfetrat M, Nikoo MH, Malekmakan L, Tabanade M. Risk factors for contrast- related acute kidney injury according to Risk, Injury, Failure, Loss and 
End- Stage criteria in patients with coronary interventions. Iranian J Kidney Dis 2010; 4: 11622.

26) Morcos SK, Thomsen HS, Webb JAW and members of contrast media safety committee of the European Society of Urogenital Radiology (ESUR). Contrast media induced nephrotoxicity: A consensus report. Eur Radiol 1999; 9: 1602- 13.

27) Schuhlen. H, Kastrati A, Dirschinger J, Hausleiter J. Intracoronary stenting and risk for major adverse cardiac events during the first month. Circulation 1998; 98: 104- 11.

28) Shulman NB, Ford CE, Hall WD. Prognostic value of serum creatinine and effect of treatment of hypertension on renal function: results from the hypertension detection and follow-up program. The Hypertension Detection and Follow-up Program Cooperative Group. Hypertension 1989; 13 (5 suppl): I80- 93.

29) Coca SG, Krumholz HM, Garg AX . Underrepresentation of renal disease in randomized controlled trials of cardiovascular disease. JAMA 2006; 296: 1377-84.

30) Chertow GM, Normand SL, McNeil BJ. "Renalism": inappropriately low rates of coronary angiography in elderly individuals with renal insufficiency. J Am Soc Nephrol 2004; 15:246268.

31) Li J-P, Momin M, Huo Y, Wang C-Y. Renal insufficiency is an independent predictor of inhospital mortality for patients with acute myocardial infarction receiving primary percutaneous coronary intervention. J Zhejiang Univ-Science B 2012; 13: 638-44.

32) Freeman RV, Mehta RH, Al Badr W, Cooper JV. Influence of concurrent renal dysfunction on outcomes of patients with acute coronary syndromes and implications for the use of glycoprotein IIb/IIIa inhibitors. J Am Coll Cardiol 2003 41: 718- 24.

33) Inrig JK, Patel UD, Briley LP, She L. Mortality, kidney disease and cardiac procedures following acute coronary syndrome. Nephrol dial Transplant 2008; 23: 934- 40.

34) Mak RH, Ikizler AT, Kovesdy CP, Raj DS. Wasting in chronic kidney disease. J Cachexia Sarcopenia Muscle 2011; 2: 9-25.

35) Wong JA, Goodman SG, Yan RT, Wald R. Temporal management patterns and outcomes of non-ST elevation acute coronary syndromes in patients with kidney dysfunction. European Heart Journal 2009; 30: 549- 57.

36) Winkelmayer WC, Charytan DM, Levin R. Poor short-term survival and low use of cardiovascular medications in elderly dialysis patients after acute myocardial infarction. AmJ Kidney Dis 2006; 47: 301-8

37) Htun P, Fateh-Moghadam S, Bischofs C, Banya W. Low responsiveness to clopidogrel increases risk among CKD patients undergoing coronary intervention. J Am Soc Nephrol 2011 22:627- 33.

38) Shlipak MG, Heidenreich PA, Noguchi H, Chertow
GM.Association of renal insufficiency with treatment and outcomes aftermyocardial infarction in elderly patients. Ann Intern Med 2002; 137:55562.

39) Diris JH, Hackeng CM, Kooman JP, Pinto YM. Impaired renal clearance explains elevated troponin $\mathrm{T}$ fragments in hemodialysis patients. Circulation 2004 109: 23- 5.

40) Jeremias A, Albirini A, Ziada KM, Chew DP. Prognostic significance of creatine kinase-MB elevation after percutaneous coronary intervention in patients with chronic renal dysfunction. Am Heart J 2002; 143: 1040- 5.

41) Gitt AK, Bauer T, Zeymer U, Zahn R. Impact of renal failure on mortality, stroke and bleeding complications in thesetting of PCI for ACS or stable angina in Europe. J Am Coll Cardiol 2010 55: $10 \mathrm{~A}$.

42) Shinohara K, Shoji T, Tsujimoto Y, Kimoto E. Arterial stiffness in predialysis patients with uremia. Kid International 2004; 65: 936- 43.

43) Appleby CE, Ivanov J, Lavi S, Mackie K. The adverse longterm impact of renal impairment in patients undergoing percutaneous coronary intervention in the drug-eluting stent era. Circ Cardiovasc Interv 2009; 2; 309- 16.

44) Huffman C, Wagman G, Fudim M, Zolty R. Reversible Cardiomyopathies-A Review. Transplantation Proceedings 2010; 42: 3673- 8.

45) Alhaj E, Alhaj N, Rahman I, Niazi TO. UremicCardiomyopathy: An Underdiagnosed Disease. Congest Heart Fail 2013; 19: E40 - E45.

46) Best PJ, berger PB, Davis BR, Grines CL. Impact of mild to moderate chronic kidney disease on the frequency of restenosis. JAm Coll Cardiol 2004; 44: 1786- 91.

47) Miyagi M, Ishii H, Murakami R, Isobe S. Impact of renal function on coronary plaque composition. Nephrol Dial Transplant 2010; 25: 175- 81.

48) Gruberg L, Dangas G, Mehran R. Clinical outcome following percutaneous coronary interventions in patients with chronic renal failure. Catheter Cardiovasc Interv 2002; 55: 66-72.

49) Kip KE, Hollabaugh K, Marroquin OC, Williams DO. The problem with composite end points in cardiovascular studies. J Am Coll Cardiol 2008; 51: 701- 7 .

50) Charytan D, Forman JP, Cutlip DE. Risk of target lesion revasularization after coronary stenting in patients with and without chronic kidney disease. Nephrol Dial Transplant 2007; 22:2578- 85.

51) Kahn JK, Rutherford BD, McConahay DR, Johnson WL. Short- and long-term outcome of percutaneous transluminal coronary angioplasty in chronic dialysis patients. Am Heart J 1990;119: 484- 9 .

52) $\mathrm{Na}$ KY, Kim CW, Song YR, Chin HJ. The association between kidney function, coronary artery disease, and clinical outcome in patients undergoing coronary angiography. J Korean Med Sci 2009; 24 (Suppl 1): S87- 94.

53) Azar RR, Prpic R, Ho KK. Impact of end- stage 
renal disease on clinical and angiographic outcomes after coronary stenting. Am J Cardiol 2000; 85: 485- 9 .

54) Verhave JC, Gansevoort RT, Hillege HL, De
Zeeuw D. Drawbacks of the use of indirect estimates of renal function to evaluate the effect of risk factors on renal function. J Am Soc Nephrol 2004;

1316-1322. 